\title{
Astrocytic proBDNF and Tonic GABA Distinguish Active versus Reactive Astrocytes in Hippocampus
}

\author{
Heejung Chun ${ }^{1,2}$, Heeyoung $\mathrm{An}^{1,2,3}$, Jiwoon Lim², Junsung Woo', \\ Jaekwang Lee ${ }^{1}$, Hoon Ryu ${ }^{4,5 *}$ and C. Justin Lee ${ }^{1,2,6 *}$ \\ ${ }^{1}$ Center for Neuroscience and Functional Connectomics, Brain Science Institute, Korea Institute of Science and Technology \\ (KIST), Seoul 02792, ${ }^{2}$ Center for Glia-Neuron Interaction, Brain Science Institute, KIST, Seoul 02792, ${ }^{3}$ KU-KIST Graduate \\ School of Converging Science and Technology, Korea University, Seoul 02841, ${ }^{4}$ Center for Neuromedicine, Brain Science \\ Institute, KIST, Seoul 02792, Korea, ${ }^{5}$ Boston University Alzheimer's Disease Center and Department of Neurology, Boston \\ University School of Medicine, Boston, MA 02118, USA, ${ }^{6}$ Division of Bio-Medical Science \& Technology, KIST School, KIST, \\ Seoul 02792, Korea
}

\begin{abstract}
Astrocytes are the most abundant cell type in the brain and they make close contacts with neurons and blood vessels. They respond dynamically to various environmental stimuli and change their morphological and functional properties. Both physiological and pathological stimuli can induce versatile changes in astrocytes, as this phenomenon is referred to as astrocytic plasticity'. However, the molecular and cellular mechanisms of astrocytic plasticity in response to various stimuli remain elusive, except for the presence of hypertrophy, a conspicuous structural change which is frequently observed in activated or reactive astrocytes. Here, we investigated differential characteristics of astrocytic plasticity in a stimulus-dependent manner. Strikingly, a stab wound brain injury lead to hypertrophy of astrocytes accompanied by increased GABA expression and tonic GABA release in mouse CA1 hippocampus. In contrast, the mice experiencing enriched environment exhibited astrocytic hypertrophy with enhanced proBDNF immunoreactivity but without GABA signal. Based on the results, we define proBDNF-positive/GABA-negative hypertrophic astrocytes as active' astrocytes and GABA-positive hypertrophic astrocytes as 'reactive' astrocytes, respectively. We propose for the first time that astrocytic proBDNF can be a bona fide molecular marker of the active astrocytes, which are distinct from the reactive astrocytes which show hypertrophy but with aberrant GABA.
\end{abstract}

Key words: Astrocytes, gamma-Aminobutyric Acid, Cell Plasticity, Wounds, Stab, brain-derived neurotrophic factor precursor

\footnotetext{
Received June 12,2018, Revised June 26,2018, Accepted June 30, 2018

* To whom correspondence should be addressed. C. Justin Lee, TEL: 82-2-958-6940, FAX: 82-2-958-6919 e-mail:cjl@kist.re.kr

Hoon Ryu, TEL: 1-857-364-5910, FAX: 1-857-364-4540 e-mail: hoonryu@bu.edu
}

\section{INTRODUCTION}

Brain plasticity is the ability of the brain to adapt to a new environment and it accompanies various morphological and functional alterations of the brain cells [1]. Among brain cells, astrocytes, which are the most abundant cell type in the mammalian brain, actively respond to various environmental stimuli and change their morphological and functional characteristics. Upon physiological or pathological stimulations, resting astrocytes undergo
Copyright (c) Experimental Neurobiology 2018. www.enjournal.org
This is an Open Access article distributed under the terms of the Creative Commons Attribution Non-Commercial License (http://creativecommons.org/licenses/by-nc/4.o) which permits unrestricted non-commercial use, distribution, and reproduction in any medium, provided the original work is properly cited. 
plastic changes in gene and protein expression, gliotransmission, and cellular morphology, which are often termed as "astrocytic plasticity" [2]. However, the molecular and cellular mechanisms of astrocytes responding to different modalities of stimulation remains elusive.

Under pathological condition, aversive experiences such as brain injury, ischemia and exposure to toxic materials such as amyloid plaques or viruses ubiquitously make reactive astrocytes [3], which have been characterized by the presence of morphological hypertrophy. In reactive astrocytes, the intermediate filament proteins in astrocytes such as glial fibrillary acidic protein (GFAP) and vimentin are increasingly expressed [3-5]. In addition to structural changes, it was reported that reactive astrocytes show switching-on of aberrant GABA production to cause a strong tonic inhibition onto neighboring neurons in hippocampal dentate gyrus (DG) of animal models of Alzheimer's disease (AD) [6,7] and in AdenoGFAP-GFP virus-treated 3D culture system [8]. At the core of the aberrant GABA production, monoamine oxidase-b (MAOB), mainly expressed in astrocytes, was shown to mediate the degradation of putrescine, a polyamine byproduct of degradation of amyloid beta $(A \beta)$ toxin, and the biosynthesis of GABA in $A \beta$ overproducing AD model of APP/PS1 mice [6]. These findings were recently recapitulated and further expanded to show sex- and age-dependent expression pattern of GABA and actions of MAO$B$ in another animal model of AD, Tg2576 mice [9]. Based on these previous studies, the astrocytic GABA has been proposed as a molecular signature of reactive astrocytes when combined with astrocytic hypertrophy.

Interestingly, astrocytes also respond to physiological stimulations under the conditions of enriched environment (EE) [10], which is a housing condition in which animals are raised in a large cage containing novel objects and running wheels to allow physical exercise and cognitive stimulations [11]. It has been reported that EE causes brain plasticity by promoting synaptogenesis [12] neurogenesis $[13,14]$, and hypertrophy and proliferation of astrocytes $[10,15]$. More importantly, we have recently demonstrated that EE causes brain plasticity by enhancing brain volume dynamics through aquaporin 4 (AQP4)-mediated water flux and volume transients in astrocytes leading to long-term plasticity, cognitive enhancement, and structural plasticity in mice and human [16]. Although EE is known to cause the plastic changes in the brain by turning-on of various genes encoding synaptic proteins and growth factors such as brain-derived neurotrophic factor (BDNF) [17], the detailed mechanism of how EE causes plastic changes in astrocytes is mostly unknown. Moreover, the observed morphological hypertrophy of astrocytic processes in EE mice [16, 18] appear to be similar to the hypertrophy in reactive astrocytes.
Despite the seemingly similar morphological characteristics, the astrocytes in EE seem to be distinct from the reactive astrocytes under brain injury condition, because $\mathrm{EE}$ is known to mitigate the inflammatory and pathological states of reactive astrocytes in ischemic $[19,20]$ and glioma [21] conditions. Therefore, it is necessary to identify the distinguishing features of the astrocytes under EE condition from those of the reactive astrocytes under various neuroinflammatory and brain injury conditions.

In this study, we set out to investigate and compare and contrast the molecular and cellular characteristics of the hypertrophied astrocytes under EE and under stab wound brain injury (STAB) condition. In the course of this study, we have newly identified that the resting astrocytes in CA1 hippocampus show robust expression of the precursor pro form of BDNF (proBDNF), but not the mature form of BDNF (mBDNF). More importantly, we identify that the hypertrophied astrocytes under EE condition show enhanced expression of proBDNF. This lead us to define the hypertrophied astrocytes under EE condition as "active" astrocytes because they are activated by physiological and environmentally beneficial stimulations. Using these recently and newly identified molecular markers, GABA for the reactive astrocytes and proBDNF for the active astrocytes, we clearly distinguish the active astrocytes as proBDNF-positive/GABA-negative and the reactive astrocytes as GABA-postive/proBDNF-negative.

\section{MATERIALS AND METHODS}

\section{Animals}

For STAB and EE conditions, 7 to 9-week-old C57BL/6J female mice were used. All animals were maintained in a vivarium with light/dark cycle (8:00 AM 8:00 PM). Animal care and handling were performed according to the directives of the Animal Care and Use Committee and institutional guidelines of KIST (Seoul, Korea). Animals were randomly selected for each group.

\section{Stab wound brain injury (STAB)}

Animals were deeply anesthetized with $2 \%$ avertin $(20 \mu \mathrm{g} g-1$, i.p.) and mounted in a stereotaxic frame. The scalp was incised and a hole was drilled into the skull above the hippocampus (AP, $-2 \mathrm{~mm}$; ML, - $1.5 \mathrm{~mm}$ from the Bregma). Then, 30-gauge needle was slowly inserted into the ipsilateral side of the brain, penetrating the hippocampus (DV $-3 \mathrm{~mm}$ from dura), left for $10 \mathrm{~min}$, and then slowly retracted. The contralateral side served as a no-injury control. Seven days after surgery, mice were sacrificed for immunostaining and electrophysiology. 


\section{Enriched environment (EE)}

Mice were randomly assigned to EE or standard condition (SC) groups and were housed in larger cages $(40 \mathrm{~cm} \times 28 \mathrm{~cm} \times 18 \mathrm{~cm})$, as previously described [16]. Only in EE group, mice were allowed access to a variety of objects of different textures and shapes including running wheels, tunnels, wood ladder and igloo in differing configurations and changed the location weekly for novelty. After 30 days, mice were sacrificed for immunostaining and electrophysiology.

\section{Immunohistochemistry}

Animals were deeply anesthetized using 2\% avertin and perfused with $0.1 \mathrm{M}$ PBS, followed by $4 \%$ paraformaldehyde solution. Brains were post-fixed in $4 \%$ paraformaldehyde at $4^{\circ} \mathrm{C}$ for 24 hrs and dehydrated in $30 \%$ sucrose at $4^{\circ} \mathrm{C}$ for 48 hrs. Brains were then cut in coronal sections of $30 \mu \mathrm{m}$ on a cryosection. Sections were blocked in $0.1 \mathrm{M}$ PBS containing $0.3 \%$ Triton X-100 (Sigma) and $2 \%$ donkey serum (Genetex) for $1 \mathrm{hr}$ at room temperature. Primary antibodies used are as following: chicken anti-GFAP (1:500, ab5541, Millipore), guinea pig anti-GABA (1:200, ab175, Millipore), chicken anti-proBDNF (1:200, ab9042, Millipore, epitope; fusion protein from mouse proBDNF containing only the prodomain region), rabbit anti-BDNF (1:50, sc20981, Santacruz, epitope; recombinant BDNF containing amino acid 130-247 from $\mathrm{N}$-terminus). The brain samples with primary antibodies were incubated overnight at $4^{\circ} \mathrm{C}$. Then, the sections were washed three times in $0.1 \mathrm{M}$ PBS and incubated in proper secondary antibodies (1:500) from the Jackson Laboratory for $3 \mathrm{hrs}$. After three rinses in 0.1 M PBS and DAPI staining at 1:10000 (PIERCE), the sections were mounted on the Polysine microscopic slide glass (Thermo Scientific). Images were acquired using a Nikon A1R confocal microscope.

\section{Image analysis and quantification}

Confocal microscopic images were analyzed using the ImageJ program (NIH). For Sholl analysis, the GFAP images were converted into binary images and the concentric circles with $1 \mu \mathrm{m}$ interval were drawn in every GFAP+ astrocytes starting from the center of the soma. Then, the sum of number of intersection crossings with each concentric circle and the ramification index, a measure of the difference in the number of intersections made in pairs of consecutive circles, were analyzed using the Sholl analysis module in ImageJ. For measuring cell density, the DAPI-positive cells were manually counted in the image area of $50 \mu \mathrm{m}^{\star} 50 \mu \mathrm{m}$ and the cell density was calculated. For measuring GABA intensity in an astrocyte, the GFAP images were converted into binary and every GFAP+ astrocyte was selected as a region of interest (ROI). Then the mean intensity value of GABA in every ROI was measured. For analyzing total GABA contents in one astrocyte, the average intensity of GABA in each GFAP+ astrocyte was multiplied by the area of GFAP-positive region. For measuring GFAP-negative GABA intensity, the soma region of interneuron was selected as a ROI and the mean GABA intensity in each ROI was measured. For localization of proBDNF or mBDNF between neuron and astrocytes, the average intensity of astrocytic proBDNF or mBDNF was measured in GFP-positive region. Then, the value were normalized by average intensity of neuronal proBDNF or mBDNF measured in CAl pyramidal layer of hippocampus.

\section{Tonic GABA measurement}

Animals were deeply anaesthetized with halothane. After decapitation, the brain was quickly excised from the skull and submerged in ice-cold cutting solution that contained (in mM): 250 sucrose, $26 \mathrm{NaHCO}_{3}, 10 \mathrm{D}(+)$-glucose, $4 \mathrm{MgCl}_{2}, 3$ myo-inositol, $2.5 \mathrm{KCl}, 2$ sodium pyruvate, $1.25 \mathrm{NaH}_{2} \mathrm{PO}_{4}, 0.5$ ascorbic acid, $0.1 \mathrm{CaCl}_{2}$, and 1 kynurenic acid, $\mathrm{pH}$ 7.4. The entire solution was continuously gassed with $95 \% \mathrm{O}_{2}-5 \% \mathrm{CO}_{2}$. The $300 \mu \mathrm{m}$ coronal brain slices containing hippocampal region were cut using a microtome and transferred to extracellular artificial cerebro-spinal fluid (ACSF) solution (in mM): $126 \mathrm{NaCl}, 24 \mathrm{NaHCO}_{3}, 1 \mathrm{NaH}_{2} \mathrm{PO}_{4}, 2.5 \mathrm{KCl}$, $2.5 \mathrm{CaCl}_{2}, 2 \mathrm{MgCl}_{2}$, and $10 \mathrm{D}(+)$-glucose, $\mathrm{pH}$ 7.4. Slices were incubated at room temperature for at least one hour prior to recording. Slices were transferred to a recording chamber that was continuously perfused with ACSF solution (flow rate $=2 \mathrm{ml} / \mathrm{min}$ ). The slice chamber was mounted on the stage of an upright Olympus microscope and viewed with a $60 \mathrm{X}$ water immersion objective lens $(\mathrm{NA}=0.90)$ with infrared differential interference contrast optics. Cellular morphology was visualized by CCD camera and Axon Imaging Workbench (Indec Systems) software. Whole-cell recordings were made from pyramidal neuron somata located in CA1 hippocampal region. The holding potential was at $-60 \mathrm{mV}$. Pipette resistance was typically $6 \sim 8 \mathrm{M} \Omega$ and the pipette was filled with an internal solution (in $\mathrm{mM}$ ): $135 \mathrm{CsCl}, 4 \mathrm{NaCl}, 0.5 \mathrm{CaCl}_{2}, 10$ Hepes, 5 EGTA, 2 Mg-ATP, $0.5 \mathrm{Na}_{2}$-GTP, 10 QX-314, pH adjusted to 7.2 with $\mathrm{CsOH}$ (278 285 mOsmol). Electrical signals were digitized and sampled at $50 \mu$ s intervals with Digidata1440A and Multiclamp 700B amplifier (Molecular Devices) using pCLAMP 10.2 software. Data were filtered at $2 \mathrm{kHz}$ with Bessel filter. The tonic GABA current was measured by baseline shift after bicuculline (50 $\mu \mathrm{M})$ applications. The amplitude and frequency of spontaneous inhibitory post-synaptic currents (sIPSCs) measured before bicuculline applications were analyzed by Minianalysis (Synaptosoft). 


\section{Statistical analysis}

The statistical significance of data for comparison was assessed by Student's two-tailed unpaired t-test between two groups and one-way ANOVA test between three or more groups. Analysis were performed with Prism (GraphPad Software, Inc.). Data distribution was assumed to be normal, but was not formally tested. Data are presented as mean \pm SEM (standard error of the mean). Levels of statistical significance are indicated as follows: ${ }^{*}(p<0.05)$,
${ }^{* *}(\mathrm{p}<0.01),{ }^{* * *}(\mathrm{p}<0.001),{ }^{* * *}(\mathrm{p}<0.0001)$.

\section{RESULTS}

\section{Aberrant GABA in hypertrophied reactive astrocytes in STAB}

To investigate the molecular and cellular alterations in reactive astrocytes, we used STAB model, which is a widely used traumatic

A

\section{Experimental Timeline}

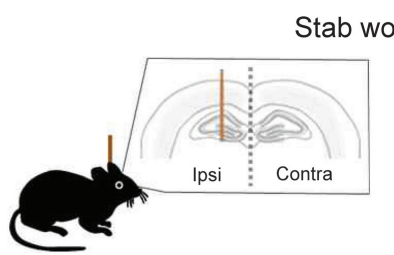

B
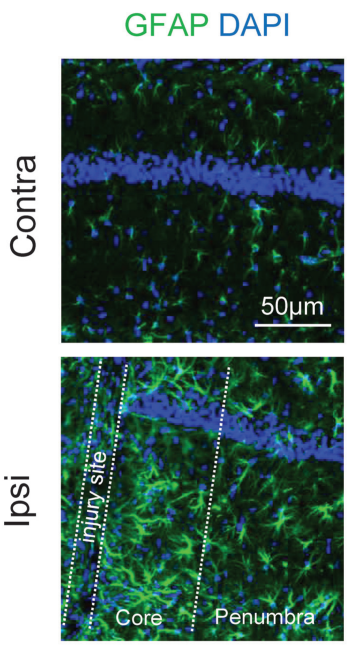

C

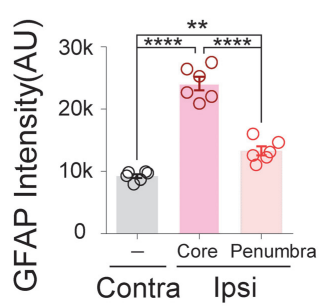

$\mathrm{D}$

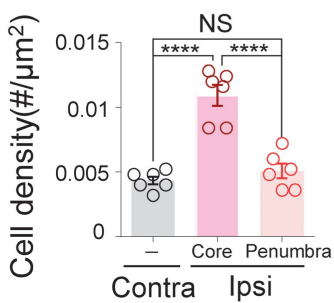

DAPI
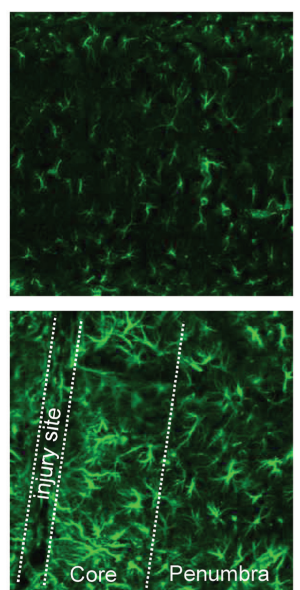

Perfusion/ 30\% Cryosection/

Fixation Sucrose Immunohistochemistry

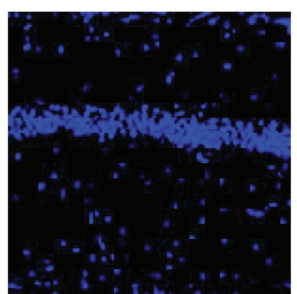

E Sholl Analysis

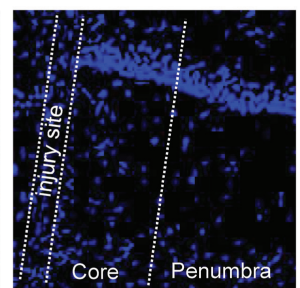

Contra

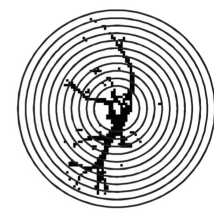

Ipsi

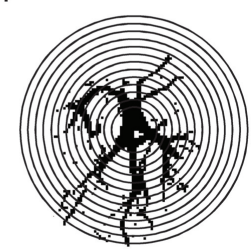

$\mathrm{F}$

G

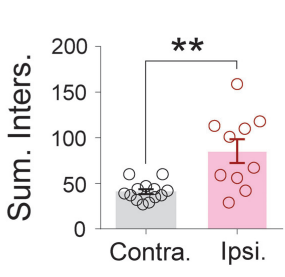

Fig. 1. The reactive astrocytes have graded reactivity in STAB. (A) Experimental timeline for stab wound injury. (B) Representative confocal images for the GFAP expression (green; GFAP, blue; DAPI) in hippocampal CA1 region of ipsilateral or contralateral side of injured brain. core; $<80 \mu \mathrm{m}$ from injury site, penumbra; $>80 \mu \mathrm{m}$ from injury site. (C) The average intensity of GFAP immunoreactivity signal in CA1 region of ipsilateral or contralateral side of hippocampus. One-way ANOVA with Tukey's multiple comparison test $\left({ }^{* *} \mathrm{p}<0.01\right.$, $\left.{ }^{* * *} \mathrm{p}<0.0001\right)$ ( $\mathrm{n}=6$ for each group). (D) Cell density in stratum radiatum of ipsilateral or contralateral side of hippocampus. One-way ANOVA with Tukey's multiple comparison test ${ }^{* * * *} \mathrm{p}<0.0001$, NS; non-significant, $\mathrm{p}>0.05$ ) ( $\mathrm{n}=6$ for each group). (E) Sholl analysis for immunostained GFAP signals. Starting radius; $3 \mu \mathrm{m}$, Interval of each concentric circles; $1 \mu \mathrm{m}$. (F) The summarized intersect of immunostained GFAP signals crossing with the concentric circles. Unpaired two-tailed-t-test $\left({ }^{* *} \mathrm{p}<0.01\right)(\mathrm{n}=13$ for Contra; $\mathrm{n}=10$ for Ipsi). (G) Ramification index of GFAP-stained astrocytic processes. Unpaired two-tailed-t-test $\left({ }^{*} \mathrm{p}<0.05\right)(\mathrm{n}=13$ for Contra; $\mathrm{n}=10$ for Ipsi). 
brain injury model with an open/penetrating head injury. Because it has been reported that astrocytic reactivity reaches a maximum level at 7-14 days after brain injury and reverses later on, as measured by increasing or decreasing GFAP expression [22, 23], we performed all experiments one week after the injury of penetrating hippocampus (Fig. 1A). We evaluated the astrocytic reactivity near the ipsilateral injured region of hippocampus and compared with control contralateral side without injury. It has been previously described that at the regions distant from the injury site, the mild and moderate reactive astrocytes are observed as having morphological hypertrophy with non-overlapping individual territory, whereas at the regions of near injury site, the severe reactive astrocytes show severe morphological hypertrophy, scar formation, and proliferative potential, with a disruption of individual territories [24]. We found that these characteristics were consistently observed near the injury site and at distant regions from the injury site (Fig. 1B). To analyze reactive astrocytes of STAB by categorizing into 'proliferating' and 'non-proliferating', we set the criteria of 80 micrometer from injury site to define 'core' and 'penumbra' based on the DAPI count in stratum radiatum of hippocampus, where GFAP-positive astrocytes are abundant (core; $<80 \mu \mathrm{m}$ from injury site, penumbra; $>80 \mu \mathrm{m}$ from injury site). Immunostaining with GFAP antibody showed that astrocytes became hypertrophied in the ipsilateral side of injured brain compared to the contralateral side, having a significantly higher GFAP intensity in the core compared to the contralateral side and the penumbral regions (Fig. 1B, C). Yet, the significantly higher GFAP intensity in the penumbral region compared to the contralateral side supports the concept of graded decrease of astrocyte's reactivity in distance from the injury site (Fig. 1B, C). Moreover, the density of cells in the core region of stratum radiatum, which is a glia-rich region, in ipsilateral CA1 hippocampus, was significantly higher compared to that in the penumbral region and in contralateral side (Fig. 1B, D). Previous reports have shown that the subset of reactive astrocytes in injured brain are proliferating, as evidenced by BrdU or Ki67 staining $[25,26]$. Therefore, the increased cells, only shown in core region of STAB, are likely to be the proliferating reactive astrocytes with severe hypertrophy, whereas reactive astrocytes in the penumbral regions are likely the non-proliferating reactive astrocytes with mild hypertrophy. To assess the detailed morphometric characteristics of the hypertrophied astrocytes, we performed Sholl analysis of astrocytes using GFAP signals (Fig. 1E). We found that there was a significantly higher sum of intersections and ramification index in penumbral regions of the ipsilateral side compared to the contralateral side (Fig. 1E G), indicating that the reactive astrocytes possess more branches and processes than the resting astrocytes.

We have previously reported that cerebellar astrocytes synthesize GABA by MAO-B [27] and the astrocytic GABA is aberrantly increased in reactive astrocytes near amyloid plaques in the DG of APP/PS1 mice [6]. To assess whether astrocytic GABA is altered in reactive astrocytes in CA1 hippocampus of injured brain, we performed immunohistochemistry with GABA and GFAP antibodies. For quantification of the astrocytic and neuronal GABA signals, we measured the average GABA intensity in GFAP-positive pixels and in GFAP-negative interneurons, respectively (Fig. 2B). We found that the GABA signal in GFAP-positive astrocytes
A

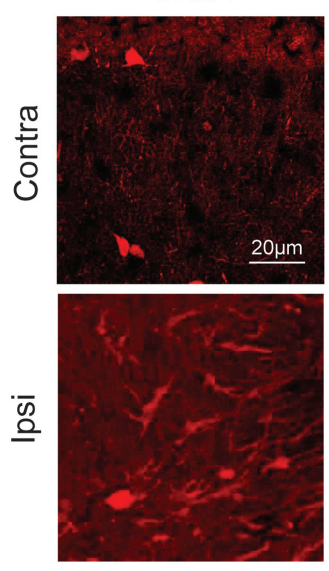

GFAP

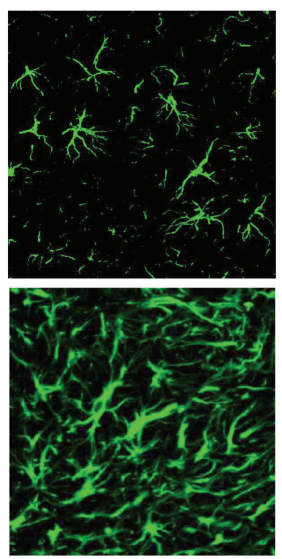

GFAP GABA

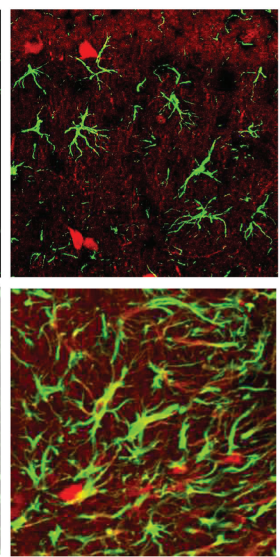

B

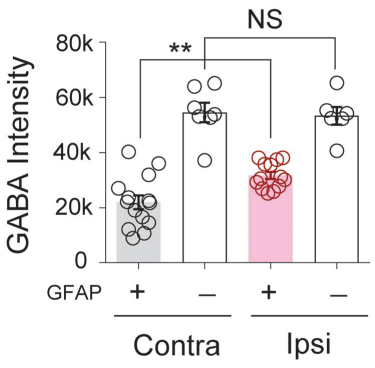

Fig. 2. Reactive astrocytes contain GABA in STAB. (A) Representative confocal images for the immunostained GABA and GFAP signals (green; GFAP, red; GABA) in hippocampal CA1 region of ipsilateral or contralateral side of injured brain. (B) Bar graph showing the comparison of the average intensity of GABA immunoreactivity in GFAP-positive and GFAP-negative/interneuron regions in CA1 region of ipsilateral or contralateral side of hippocampus of stab wound injured mice. Unpaired two-tailed-t-test ${ }^{* *} \mathrm{p}<0.01$, NS; non-significant, $\left.\mathrm{p}>0.05\right)(\mathrm{n}=14$ for GFAP+ in Contra; $\mathrm{n}=7$ for GFAP- in Contra; $\mathrm{n}=13$ for GFAP+ in Ipsi; $\mathrm{n}=6$ for GFAP- in Ipsi). 
was significantly increased in the ipsilateral side of the injury compared to the contralateral side, whereas neuronal GABA signals were not altered (Fig. 2A, B). These findings provide the evidence for the aberrant GABA in reactive astrocytes of penetrating brain injury.

\section{Aberrant tonic GABA current in STAB}

We have previously demonstrated that high intracellular GABA content in cerebellar astrocytes can be released tonically through Best 1 channel and detected in neighboring granule neurons in the form of tonic GABA current [28]. Furthermore, we reported that unlike the cerebellar astrocytes, hippocampal astrocytes do not contain GABA and the tonic GABA current is also much lower in the pyramidal neurons of hippocampal CA1 compared to the granule neurons of cerebellum [29]. Lastly, we reported that the aberrant GABA in reactive astrocytes of the DG region of hippocampus is released and detected as tonic GABA current in the dentate granule neurons of APP/PS1 mice [6, 7]. Thus, we hypothesized that the tonic GABA release from reactive astrocytes in STAB should be elevated and detected as an enhanced tonic GABA current in CAl pyramidal neurons. To test this idea, we performed slice patch clamp recordings to record the tonic GABA current from CAl pyramidal neurons of the ipsilateral and contralateral hippocampus of STAB mice (Fig. 3A) and measured the $\mathrm{GABA}_{\mathrm{A}}$ antagonist, bicuculline-sensitive current. We found a significantly higher tonic GABA current in ipsilateral side compared to the contralateral side of STAB mice (Fig. 3B, C). In contrast, the amplitude and frequency of spontaneous inhibitory post-synaptic currents (sIPSCs) were not significantly altered (Fig. 3B, D, E), indicating that phasic or synaptic GABA is not altered. These results imply that the aberrant GABA in reactive astrocytes is tonically released and strongly inhibits the neighboring neurons.

\section{A Experimental Timeline}

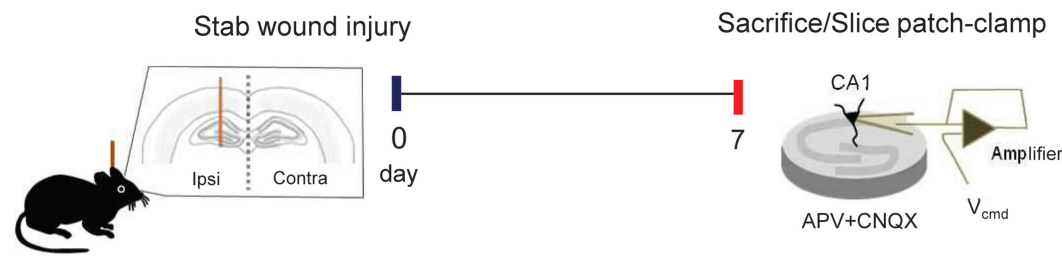

B

Contra

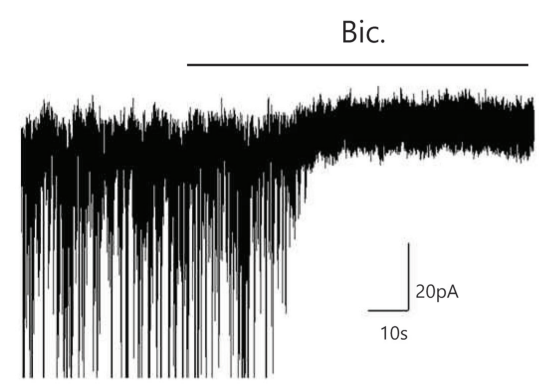

Ipsi

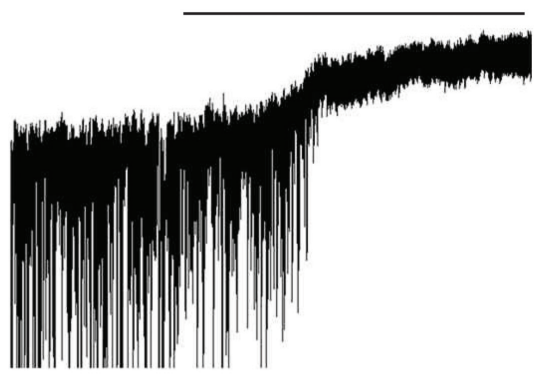

C

D

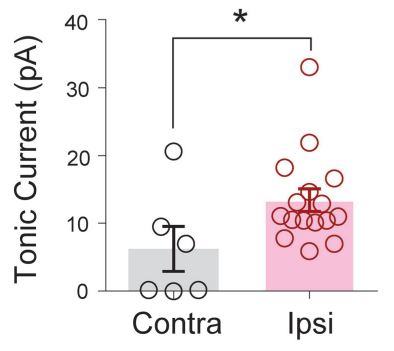

E

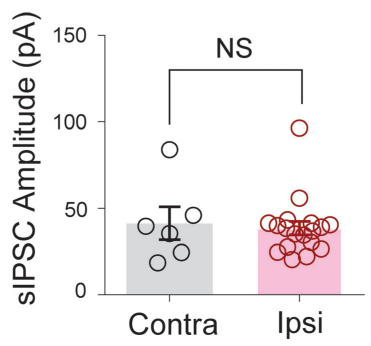

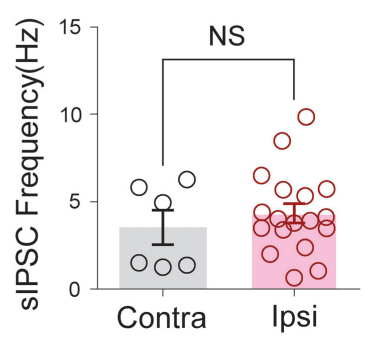

Fig. 3. Tonic GABA current is high near the injury site. (A) Schematic illustration for tonic current recording in hippocampal CAl pyramidal neurons by whole-cell patch clamp and experimental timeline for stab wound injury. (B) Representative traces of $\mathrm{GABA}_{\mathrm{A}}$ receptor-mediated current recorded from hippocampal CA1 pyramidal neurons in injured mice. Bic. indicates the adding of $50 \mu \mathrm{M}$ bicuculline in the recording solution. (C) The amplitude of tonic current ( $\mathrm{n}=6$ for Contra; $\mathrm{n}=16$ for Ipsi). (D) The amplitude of sIPSCs before bicuculline applications $(\mathrm{n}=6$ for Contra; $\mathrm{n}=18$ for Ipsi). (E) The frequency of sIPSCs before bicuculline applications. $(\mathrm{n}=6$ for Contra; $\mathrm{n}=18$ for Ipsi). Unpaired two-tailed-t-test (NS; non-significant, $\left.{ }^{*} \mathrm{p}<0.05, \mathrm{p}>0.05\right)$. 
A

Cage Settings

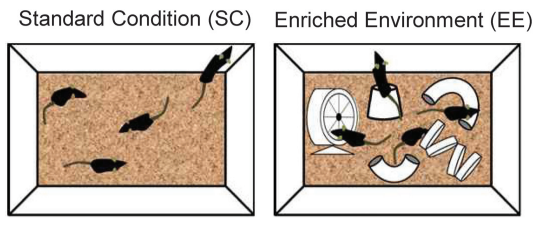

C

GFAP GABA DAPI
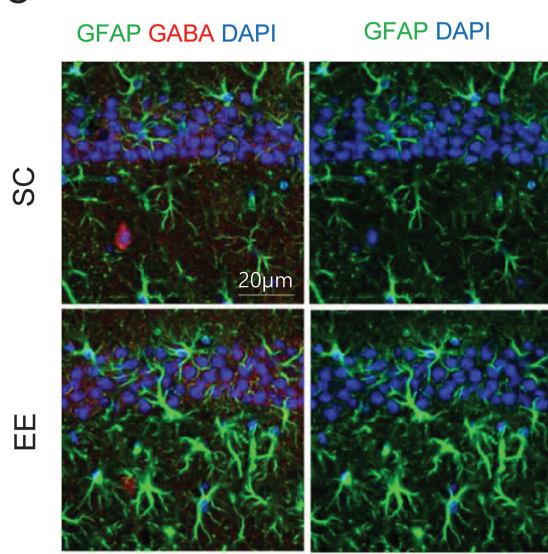

E

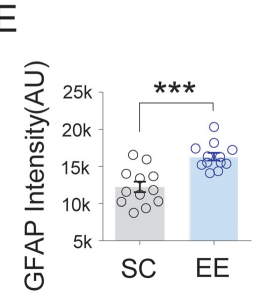

$\mathrm{F}$

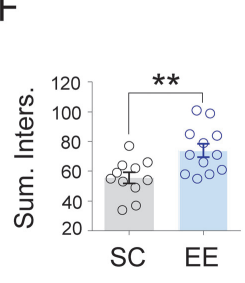

B

Experimental Timeline

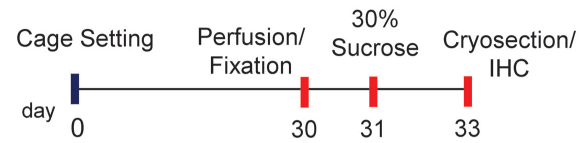

Fig. 4. Aberrant GABA is absent in hypertrophied active astrocytes of EE. (A) Schematic diagram of enriched environment. (B) Experimental procedures for histology in enriched environment (EE). (C) Representative confocal images for the immunostained GABA and GFAP signals (green; GFAP, red; GABA, blue; DAPI) in hippocampus of mice in EE. (D) Sholl analysis for immunostained GFAP signals. Starting radius; $3 \mu \mathrm{m}$, Interval of each concentric circles; $1 \mu \mathrm{m}$. (E) The average intensity of GFAP immunoreactivity signals in CAl region of EE or SC hippocampus. ( $\mathrm{n}=12$ for $\mathrm{SC} ; \mathrm{n}=12$ for $\mathrm{EE})$. (F) The summarized intersect of immunstained GFAP signals crossing with the concentric circles following Sholl analysis ( $\mathrm{n}=11$ for $\mathrm{SC} ; \mathrm{n}=12$ for EE). (G) Ramification index of GFAP-stained astrocytic processes $(n=10$ for SC; $n=12$ for EE). (H) Quantification of GABA intensity in GFAP-positive- or GFAPnegative- pixels in standard and EE conditions. Unpaired two-tailed-t-test $\left({ }^{* *} \mathrm{p}<0.01\right.$, ${ }^{* * *} \mathrm{p}<0.001$, NS; non-significant, $\left.\mathrm{p}>0.05\right)$ $(\mathrm{n}=15$ for GFAP+ in SC; $\mathrm{n}=7$ for GFAP- in $\mathrm{SC} ; \mathrm{n}=17$ for GFAP+ in EE; $\mathrm{n}=7$ for GFAP- in $\mathrm{EE})$.

\section{Similar level of astrocytic GABA and tonic GABA current between standard and enriched condition}

It has been reported that resting astrocytes can become hypertrophied under EE or after intense physical exercise [10, 30]. Therefore, we tested whether the hypertrophied astrocytes under EE contain aberrant GABA or not. Next, after housing the mice in either EE or SC, we performed immunohistochemistry with GFAP and GABA antibodies in CA1 hippocampus. As previously reported [16], astrocytes in EE mice became hypertrophied as evidenced by a significant increase in GFAP intensity (Fig. 4C, E), sum of intersections (Fig. 4D, F), and ramification index (Fig. 4D, G). Remarkably, there was no sign of aberrant GABA in the hypertrophied astrocytes under EE condition as evidenced by the similar low level of GABA intensity in GFAP positive cells between EE and SC conditions (Fig. 4H). The level of GABA intensity in GFAP-negative cells, presumably representing neuronal GABA, was also similar between EE and SC conditions (Fig. 4H). Next, we compared the amount of astrocytic GABA between STAB and EE.
Additionally, we separately analyzed the astrocytic GABA in core and penumbra of ipsilateral side of STAB. For measuring GABA contents in astrocytes, we summed up the intensity of GABA in each pixel in GFAP-positive area. As a result, we consistently observed that astrocytic GABA amount was aberrantly increased in STAB model, but not in EE model (Fig. 5). Interestingly, we observed more GABA contents in core region than in penumbra region of ipsilateral side, implicating the graded reactivity of astrocytes in STAB (Fig. 5). Additionally, we found that the GABA contents in contralateral side of STAB were significantly higher than those in SC and EE conditions (Fig. 5), which might be affected from injured hemisphere. Conclusively, these results indicate that the aberrant GABA distinguishes the hypertrophied reactive astrocytes from the hypertrophied active astrocytes and its amounts can represent the gradual reactivity of astrocytes in STAB.

To see if the absence of aberrant GABA correlates well with an absence tonic GABA current in CA1 pyramidal neurons, we performed slice patch clamp in hippocampus of EE and SC mice 


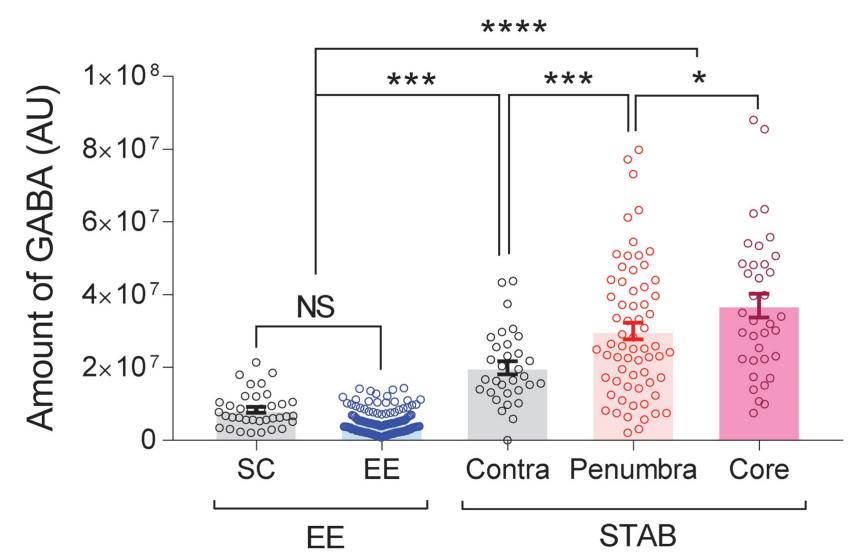

Fig. 5. Comparison of astrocytic GABA contents between active and reactive astrocytes. Quantification and comparison of GABA contents in GFAP-positive regions in EE and STAB conditions. One-way ANOVA with Tukey's multiple comparison test $\left({ }^{*} \mathrm{p}<0.05,{ }^{* * *} \mathrm{p}<0.001,{ }^{* * *} \mathrm{p}<0.0001\right.$, $\mathrm{NS}$; non-significant, $\mathrm{p}>0.05)(\mathrm{n}=36$ for $\mathrm{SC} ; \mathrm{n}=142$ for $\mathrm{EE} ; \mathrm{n}=32$ for contra; $n=65$ for penumbra; $n=36$ for core).
(Fig. 6A). We found a similar low level of tonic GABA current in EE compared to SC condition (Fig. 6B, C). The amplitude and frequency of sIPSC were also not distinguishable between SC and EE conditions (Fig. 6B, D, E). These results indicate that the absence of aberrant GABA can be a useful marker for the hypertrophied active astrocytes in EE mice. Therefore, based on these findings we can safely define the GABA-negative and hypertrophied astrocytes as "active" astrocytes and these active astrocytes are activated or stimulated by non-aversive, environmentally beneficial stimulations.

\section{proBDNF, but not $\mathrm{mBDNF}$, is expressed in resting astrocytes of CA1 hippocampus}

If the aberrant GABA is a positive molecular marker for the reactive astrocytes, what can be a positive molecular marker for the active astrocytes? It has been previously reported that growth factors such as nerve growth factor (NGF) and BDNF are enhanced in EE [31-33]. However, whether astrocytes express endogenous BDNF is unknown, although astrocytes have been shown to uptake and recycle exogenously introduced proBDNF [34]. Thus, we first ex-

\section{A}

Experimental Timeline

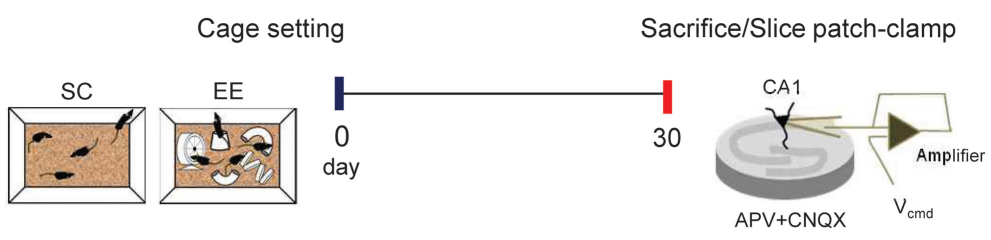

B

SC

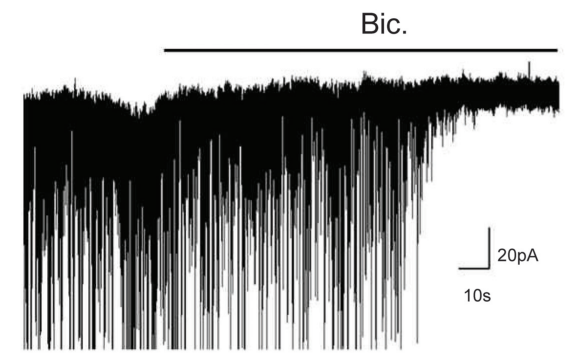

C

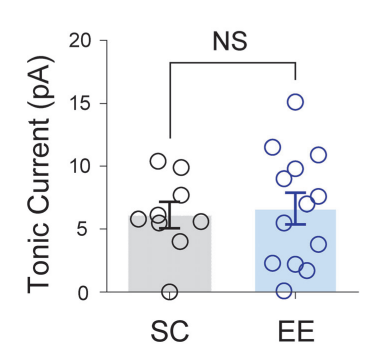

EE

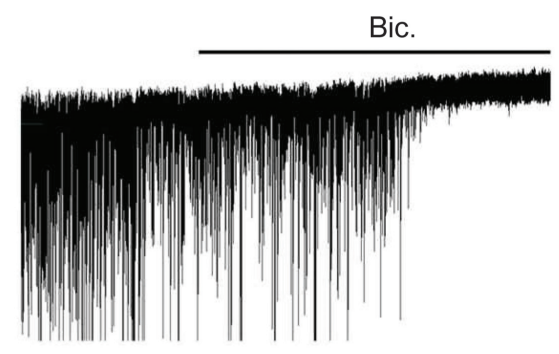

E

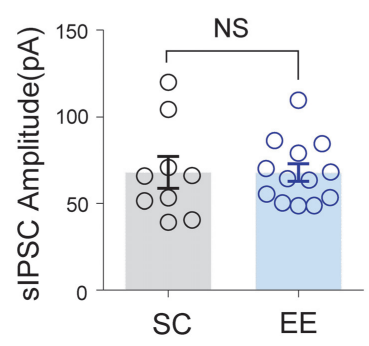

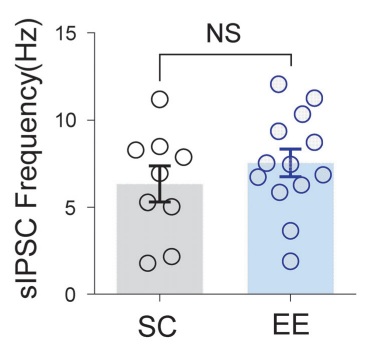

Fig. 6. Low level of tonic GABA current in EE. (A) Schematics and experimental procedures for tonic GABA measurement in mice in enriched or standard environment. (B) Representative traces of $\mathrm{GABA}_{\mathrm{A}}$ receptor-mediated current recorded from hippocampal CA1 pyramidal neurons from mice in EE. Bic indicates the adding of 50 $\mu \mathrm{M}$ bicuculline in the recording solution. (C) The amplitude of tonic current $(\mathrm{n}=10$ for SC; $\mathrm{n}=12$ for EE). (D) The amplitude of sIPSCs before bicuculline applications ( $\mathrm{n}=9$ for SC; $n=13$ for EE). (E) The frequency of sIPSCs before bicuculline applications. Unpaired two-tailed-t-test (NS; non-significant, $\mathrm{p}>0.05)(\mathrm{n}=9$ for $\mathrm{SC} ; \mathrm{n}=13$ for $\mathrm{EE})$. 
amined whether resting astrocytes express proBDNF, mBDNF or both by using commercially available antibodies against proBDNF and BDNF. We found a robust expression of proBDNF in GFAPpositive resting astrocytes of CA1 (Fig. 7A, B) and DG (Fig. 8A) of hippocampus. In contrast, we found minimal expression of mBDNF in astrocytes, whereas GFAP-negative cells show mBDNF expression (Fig. 7C, D). Next, we examined whether the level of astrocytic proBDNF was altered in active astrocytes of $\mathrm{EE}$ and in reactive astrocytes of STAB. Interestingly, we found that proBDNF signals in the molecular layer of DG, where most proBDNFpositive cells were GFAP-positive astrocytes (Fig. 8A), were significantly increased in EE compared to SC condition (Fig. 8B D). In marked contrast, the proBDNF level in GFAP-positive astrocytes was not increased in STAB, rather reduced in the core region of STAB (Fig. 9). These results support the idea that proBDNF can be a useful molecular marker for the active astrocytes.
A
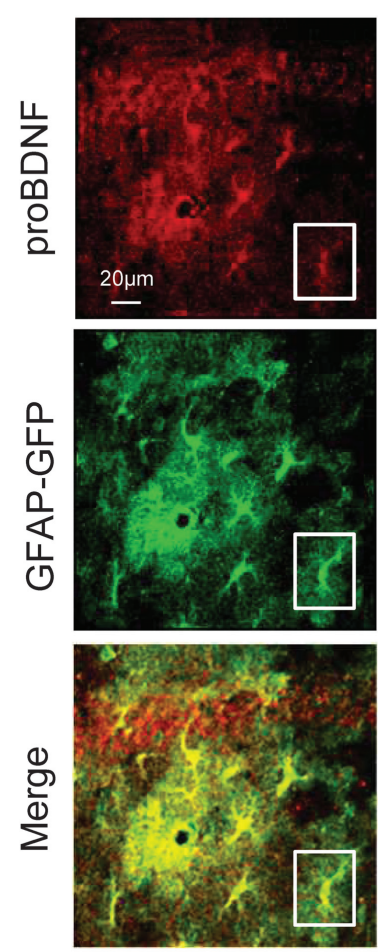

B

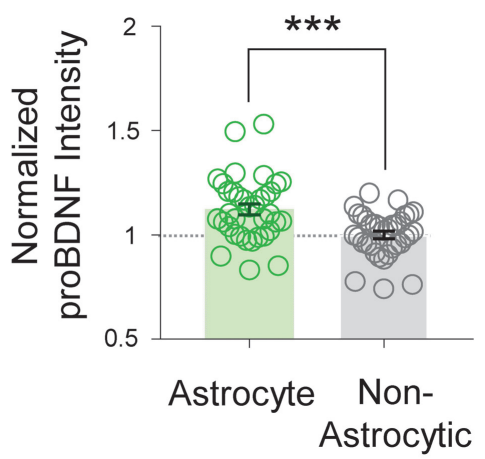

C
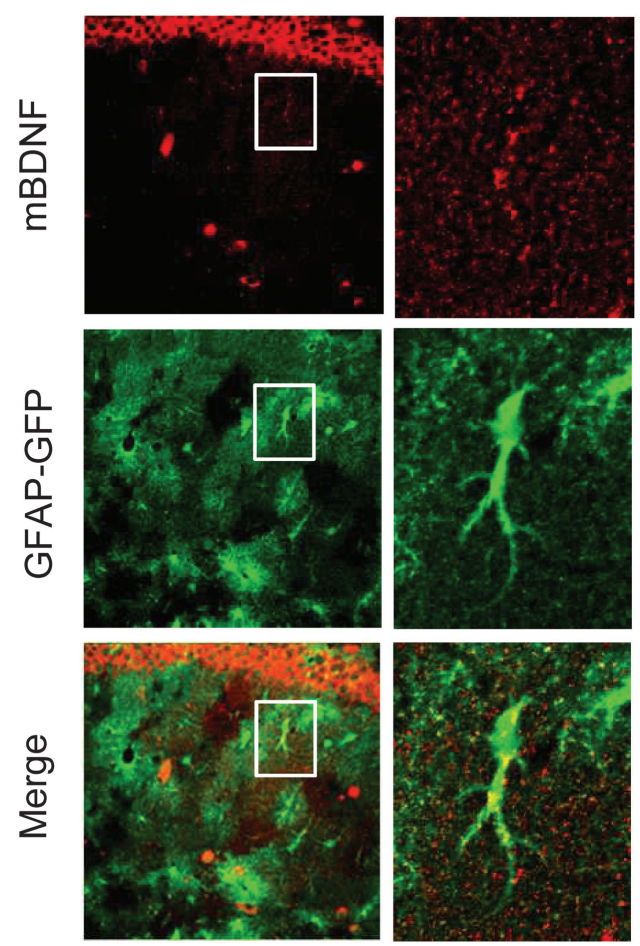

D

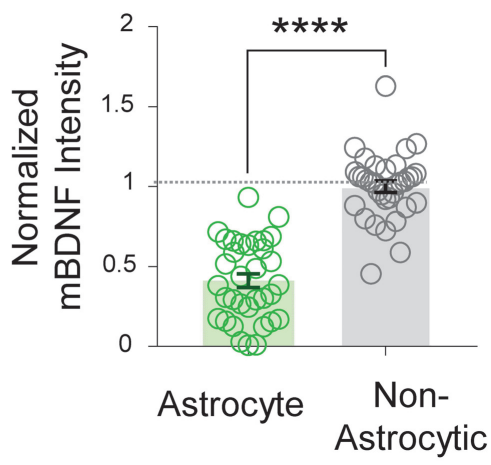

Fig. 7. The expression of proBDNF and mBDNF in resting astrocytes of hippocampus. (A) Representative confocal images for the immunostained proBDNF in CA1 hippocampus of GFAP-GFP mice (red; proBDNF, green; GFP). (B) Bar graph showing the normalized average intensity of astrocytic and non-astrocytic proBDNF in GFAP-GFP mice. Unpaired two-tailed-t-test $\left.{ }^{* * *} \mathrm{p}<0.001\right)(\mathrm{n}=34$ for Astrocyte; $\mathrm{n}=34$ for Neuron). (C) Representative confocal images for the immunostained mBDNF in CA1 hippocampus of GFAP-GFP mice (red; mBDNF, green; GFP). (D) Bar graph showing the normalized average intensity of astrocytic and non-astrocytic mBDNF in GFAP-GFP mice. Unpaired two-tailed-t-test $\left.{ }^{* * * *} \mathrm{p}<0.0001\right)(\mathrm{n}=36$ for Astrocyte; $\mathrm{n}=35$ for Neuron). 
A
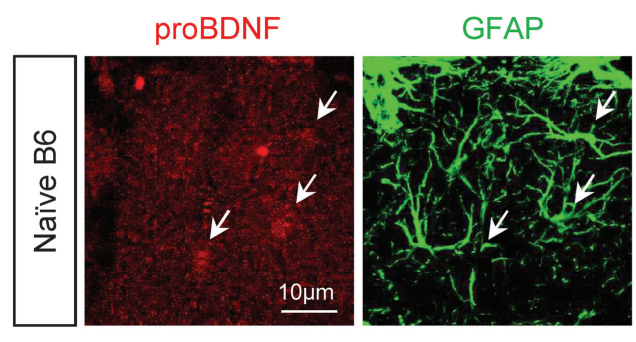

C

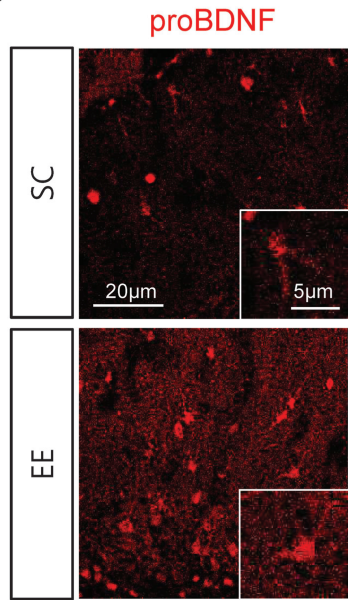

proBDNF DAPI

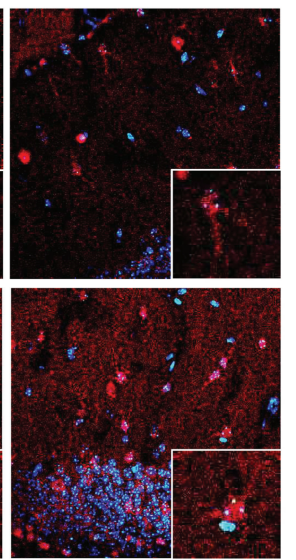

B

\section{Experimental Timeline}
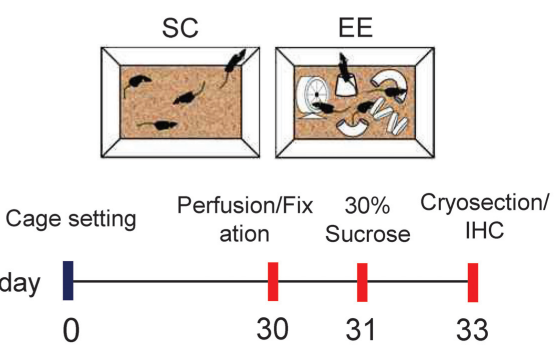

$\mathrm{D}$

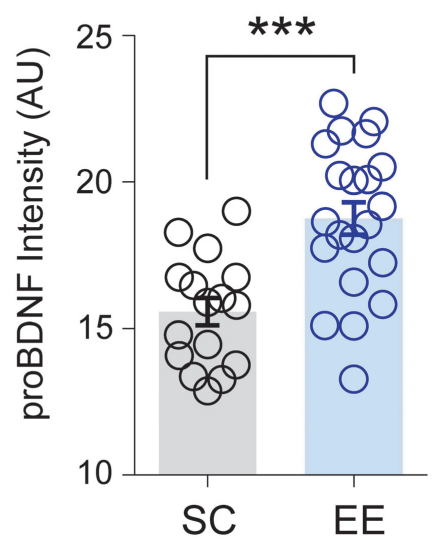

Fig. 8. The level of proBDNF is enhanced in EE. (A) Expression of proBDNF in GFAP-positive astrocytes at molecular layer of DG hippocampus (red; proBDNF, green; GFAP). (B) Experimental timeline for histology in enriched environment (EE). (C) Representative confocal images for the immunostained proBDNF (red; proBDNF, blue; DAPI) in DG hippocampus in EE or SC mice. (D) Bar graph showing the comparison of the average intensity of proBDNF immunoreactivity in molecular layer of DG. Unpaired two-tailed-t-test $\left.{ }^{(* *} \mathrm{p}<0.001\right)(\mathrm{n}=16$ for SC; $\mathrm{n}=21$ for EE).

\section{DISCUSSION}

This study is the very first attempt to categorize the hypertrophied astrocytes into active and reactive astrocytes based on the newly identified molecular markers: aberrant GABA and proBDNF (Fig. 10). We have demonstrated that resting astrocytes transform into active astrocytes under the conditions of nonaversive, environmentally beneficial stimulations such as high neuronal and synaptic activities, physical exercise, and EE, whereas resting astrocytes transform into reactive astrocytes under the conditions of aversive stimulations such as traumatic brain injury, toxin challenges and viral infections, which usually accompany neuroinflammation (Fig. 10). In regards to active astrocytes, we have previously shown that intense neuronal activities and repeated experiences cause astrocytic volume transients that possibly lead to a release of growth factors such as proBDNF, which eventually results in brain plasticity and enhanced cognition [16]. On the other hand, reactive astrocytes are abnormally exposed to toxic materials including chemicals and protein aggregates which need to be taken up and digested by astrocytes $[35,36]$. The digested toxic materials are degraded to polyamines such as putrescine, which is further degraded into GABA by MAO-B enzyme [6]. The aberrantly produced GABA is released tonically and strongly exert an inhibition of neuronal activity [6]. It is highly possible that in both active and reactive astrocytes the reactive oxygen species are excessively produced by high metabolic demand in active astrocytes and by MAO-B in reactive astrocytes $[37,38]$. The excessive ROS probably induces hypertrophy by downstream mechanisms $[38,39]$ (Fig. 10). This new model of active and reactive astrocytes are supported by the newly identified markers, aberrant GABA and proBDNF, and the proposed downstream mechanisms need to be validated in the future experiments.

What is the link between astrocytic GABA and hypertrophy? This question can be explained if we consider the mechanisms to 
A

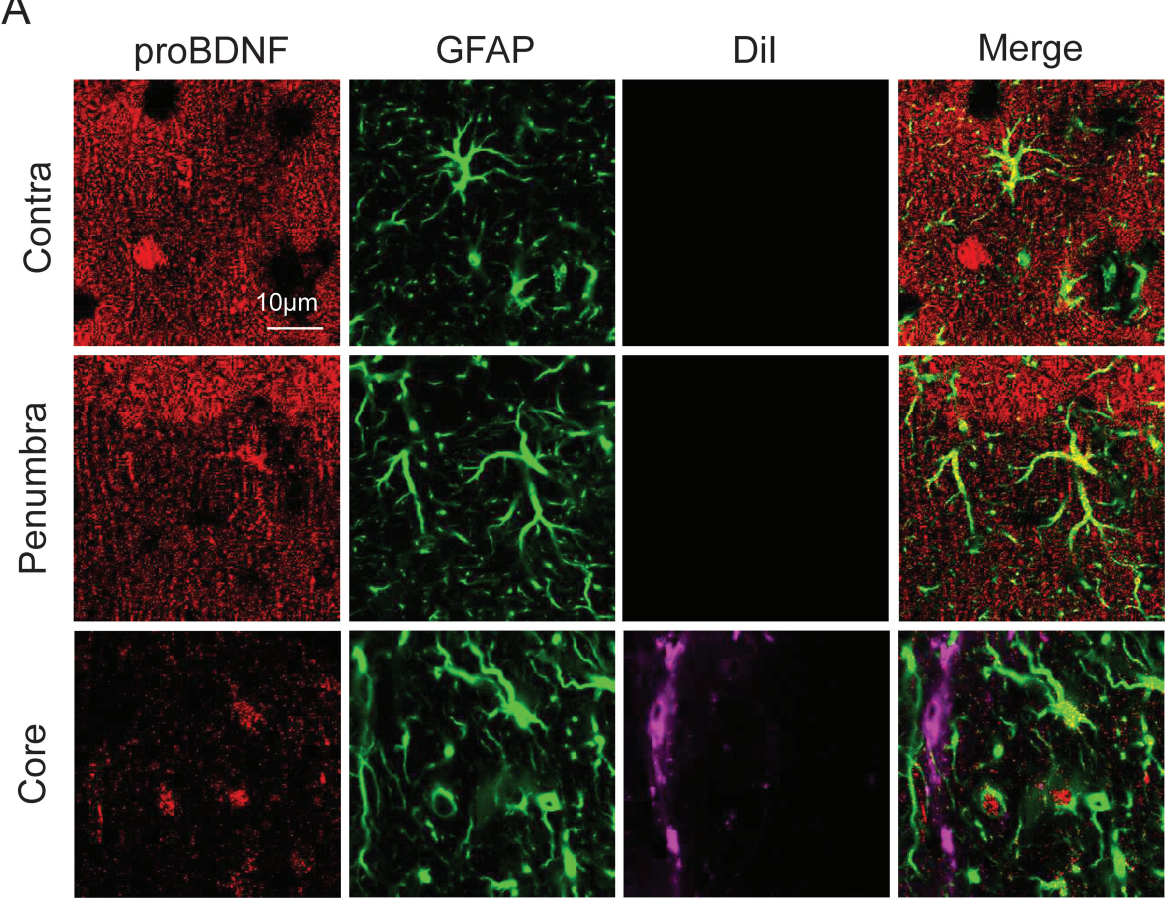

\section{B}

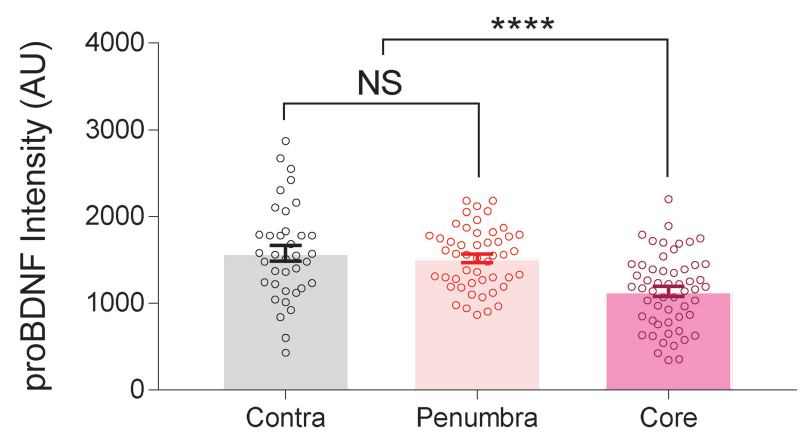

Fig. 9. The level of proBDNF is not increased in STAB. (A) Representative confocal images for the immunostained proBDNF (red; proBDNF, green; GFAP, magenta; DiI indicating injury site) in CA1 hippocampus of STAB mice. (B) Bar graph showing the comparison of the average intensity of proBDNF immunoreactivity in GFAP-positive pixels in STAB mice. One-way ANOVA with Tukey's multiple comparison test $\left({ }^{* * * *} \mathrm{p}<0.0001, \mathrm{NS}\right.$; non-significant, $\mathrm{p}>0.05)(\mathrm{n}=37$ for contra; $\mathrm{n}=49$ for penumbra; $\mathrm{n}=55$ for core).

increase astrocytic GABA in reactive astrocytes. In AD model, it is previously suggested that amyloid beta toxin can be degraded into putrescine to produce GABA in astrocytes [6]. In this study, both amyloid beta and putrescine consistently increase the release of GABA from astrocytes, which was measured in GABA sensor cells. Moreover, these phenomenon were significantly impaired by $\mathrm{MAO}-\mathrm{B}$ inhibitor selegiline. It implicates that, at least in AD model, MAO-B dependent astrocytic GABA pathway is activated to degrade toxic materials. Interestingly, MAO-B inhibition in APP/PS1 mice by selegiline significantly reduces the hypertrophy of reactive astrocytes near amyloid plaques [6] and MAO-B overexpression in astrocytes markedly induce the hypertrophy of astrocytes [38]. In other reports, it has been shown that ROS induces hypertrophy of astrocytic processes [38,39] as well as cell proliferation [40] via intracellular signaling pathways. Considering the fact that MAO-
$B$ action produces ROS by oxidizing monoamine such as acetylputrescine, it is highly likely that MAO-B-mediated ROS can induce hypertrophy in reactive astrocytes during the process of degradation of toxic materials. Moreover, the excessive activation of this ROS-producing pathway might induce astrocyte proliferation, as shown in the core of STAB, even though GABA is reported to inhibit DNA synthesis [41]. In the stab-wound brain-injury model, it could be postulated that cellular debris as a by-product of brain injury might be taken up by astrocytes and degraded to produce metabolites such as putrescine to activate MAO-B [42] Based on these new insights, the graded property of reactive astrocytes could be reflected by astrocytic hypertrophy and the level of astrocytic GABA, which should be proportional to the extent of toxic materials or the severity of injury, as shown in the injured brain (Fig. 1B, F and Fig. 5) and in the brain of APP/PS1 mice [6] 

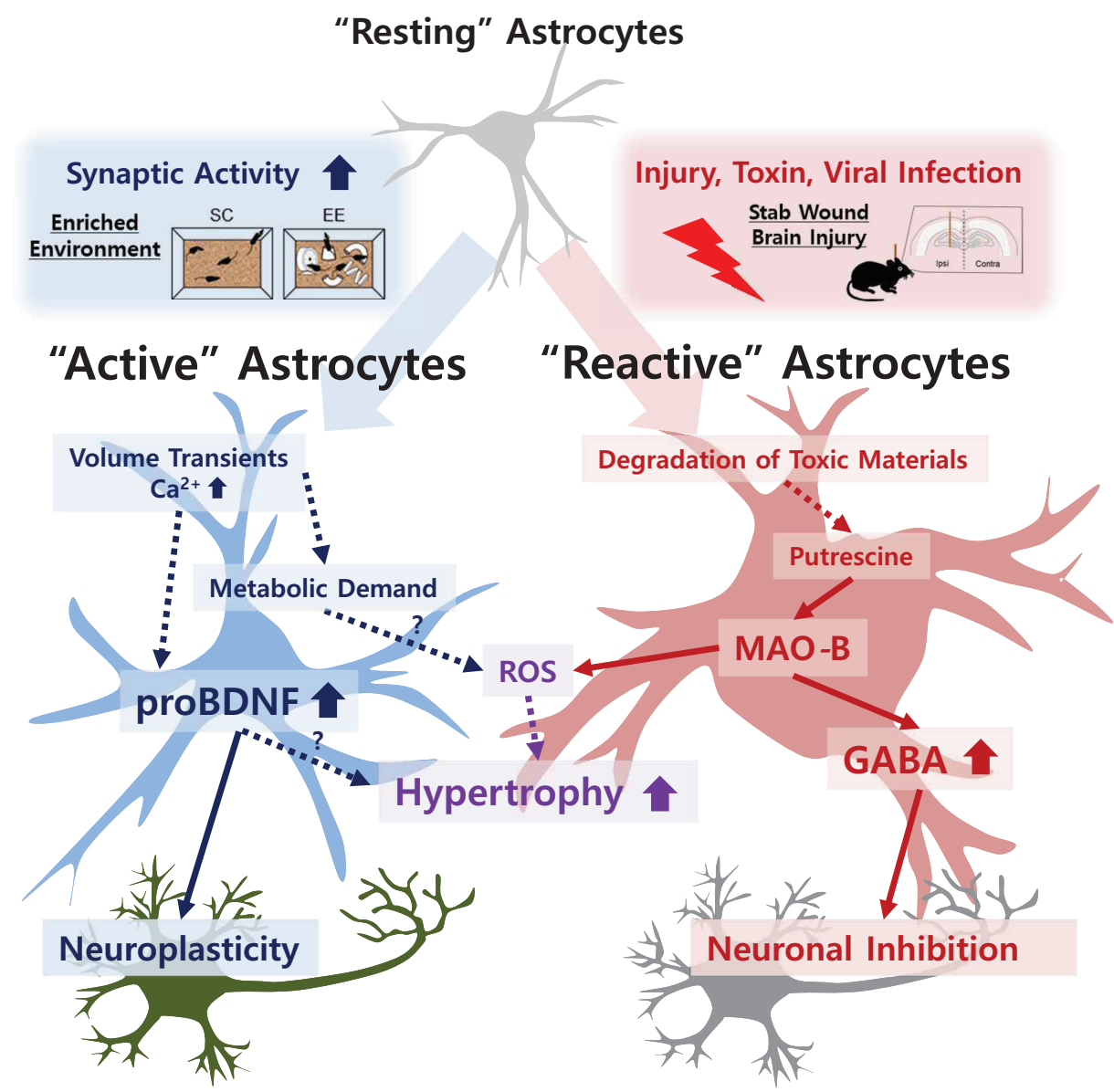

Fig. 10. Hypothetical schematic diagram between "reactive" astrocytes and "active" astrocytes.

According to the experimental observations from our group, we found MAO-B-dependent astrocytic GABA increase in motor cortex of capsular infarct model and in spinal cord of spinal cord injury model, where we also observed the hypertrophy of astrocytes. Conclusively, these implicate that astrocytic hypertrophy and GABA increase are a general mechanism for reactive astrocytes. Furthermore, this novel concept of the aberrant astrocytic GABA as the result of an active degradative process of toxic materials in STAB needs to be investigated in the future.

Then, how does EE induce astrocytic hypertrophy in the absence of GABA production? It has been reported that the brain volume, the capillary volume and the rate of respiratory energyconsumption are increased in EE $[16,37,43]$. Especially, the activity of mitochondrial complex I, which is a large enzyme catalyzing the first step of the mitochondrial electron transport chain, was found to be increased by about $80 \%$ in rat brain of EE, compared to standard conditions [37]. We hypothesized that EE might put more sensory or synaptic inputs to brains and these might increase volume transients and metabolic demand in astrocytes. We have previously demonstrated that EE increases the intrinsic optical signals, which represent the increase of astrocytic volume transients and calcium signals in astrocytes [16]. These might increase the synthesis of growth factors such as proBDNF (Fig. 8) and the various metabolites through respiratory reaction, resulting in ROS production. These possible pathways could lead to ROS-mediated hypertrophy of astrocytes without GABA increase. These distinct pathways in active astrocytes should be independent of GABA production pathway which is optimized for degradation of toxic materials in reactive astrocytes. These exciting possibilities awaits future investigations.

In general, astrocytic morphology is linked to function: the more branching, the more $\mathrm{Ca}^{2+}$ and synaptic activities. In the EE condition, we found that astrocytes in EE and STAB have more branching based on Sholl analysis. Sholl analysis measures the degree of branching by finding the number of intersection when a concentric circles are drawn. We measured the sum of intersect and the ramification index in both EE and STAB (penumbra region) conditions and found that the degree of branching is increased 
to the similar level in EE and STAB conditions. This suggests that the measure of branching by Sholl analysis is not a good distinguishing indicator between the two conditions, active and reactive astrocytes. However, the fine detail of the branching pattern and geometric features of the fine processes are not well examined by Sholl analysis. A detailed, in-depth image analysis, perhaps guided by the newly available deep-learning algorithms, is required to fully characterize the distinguishing morphological features for each type of astrocytes. This exciting possibility of morphological analysis of each astrocyte type awaits future investigations.

Although there has been a few pieces of evidence supporting the presence of astrocytic release of neurotrophic factors [44], most of the studies on BDNF have focused on neuronal cells. In current study, we firstly compared the cellular expression and localization of proBDNF and mBDNF using commercially available antibodies in CA1 hippocampus. Remarkably, proBDNF was detected in astrocytes rather than neurons (Fig. 7A, B), whereas mBDNF was minimally expressed in astrocytes but majorly detected in neuronal cells (Fig. 7C, D), as previously reported [45]. These results raise several possibilities. First, the proBDNF might be majorly synthesized in astrocytes [46] and released from astrocytes into extracellular space. Second, the released proBDNF in extracellular space is processed into mature form of BDNF by the tissue plasminogen activator/plasmin system [47] and the processed mature form of BDNF might be mainly taken up by neurons. Third, as previously described [34], the neuronal proBDNF might be released, taken up and stored by astrocytes, and recycled to be processed into mature form. These interesting possibilities need to be tested in the future. Depending on whether the proBDNF is synthesized or taken up by astrocytes, the precise mechanism of how astrocytic proBDNF is enhanced in EE can be explained.

We have detected the higher tonic GABA current in injured brain, which correlated well with the aberrant increase in GABA content in reactive astrocytes. In contrast, there was no detectable increase of tonic GABA currents in EE, which also correlated well with the low GABA content in active astrocytes. We have previously reported this strong correlation between astrocytic GABA content and tonic GABA current in cerebellum and hippocampus [29]. We reported that in cerebellum a high level of astrocytic GABA expression is correlated with a large tonic GABA current of about $\sim 25 \mathrm{pA}$, whereas in hippocampus CA1 a minimal GABA expression in astrocytes correlated well with the lack of tonic GABA current [29]. Moreover, we observed no change of synaptic GABA both in STAB and in EE by measuring amplitude and frequency of sIPSC (Fig. 3D, E and Fig. 6D, E). These are consistent with the immunostaining data showing no change of neuronal GABA expression (Fig. 2B and Fig. 4H). Therefore, the immunohistochemi- cal method of staining GABA can be an excellent marker for the extent of tonic GABA and synaptic GABA release.

What can be the function of astrocytic GABA and astrocytic proBDNF? The increased tonic GABA current in STAB could cause a strong neuronal inhibition, as shown in animal model of $\mathrm{AD}$ [6], whereas there might be no neuronal inhibition by tonic GABA in EE with low level of tonic GABA current. Instead, EE increased the expression of proBDNF (Fig. 8C, D) and the increased release of proBDNF from astrocytes should increase neuronal excitability, synaptogenesis and synaptic plasticity [48]. Conversely, the proBDNF increase was not observed in STAB but rather reduced in the core region of ipsilateral side of STAB (Fig. 9), where aberrant GABA might suppress proBDNF expression as previously reported [49]. These reciprocal relationship between the two molecular markers, proBDNF and GABA, strongly solidify our categorization of the hypertrophied astrocytes. Moreover, these should help to understand the functional diversity of astrocytes beyond the regional and morphological diversity of astrocytes [50]. In summary, the novel concept of distinguishing active versus reactive astrocytes using novel molecular markers, aberrant GABA and proBDNF, is an important step forward in the study of astrocytes. These new concepts should be further investigated for in-depth analysis and characterization of the astrocytic responses and plasticity. These attempts should help us gain further insights towards understanding the multifaceted and complex roles of astrocytes in the brain.

\section{ACKNOWLEDGEMENTS}

This research was supported by Creative Research Initiative Program, Korean National Research Foundation (NRF) (2015R1A3A2066619), the Brain Research Program through NRF funded by the Ministry of Science and ICT (2018M3C7A1056682) and the Grant (2E28411) from Korea Institute of Science and Technology (KIST) (CJL). This study was also supported by the NRF-2016M3C7A1904233 and the Grant (2E26663) from KIST (HR).

\section{REFERENCES}

1. Shaffer J (2016) Neuroplasticity and clinical practice: building brain power for health. Front Psychol 7:1118.

2. Pirttimaki TM, Parri HR (2013) Astrocyte plasticity: implications for synaptic and neuronal activity. Neuroscientist 19:604-615.

3. Ben Haim L, Carrillo-de Sauvage MA, Ceyzériat K, Escartin C (2015) Elusive roles for reactive astrocytes in neurodegen- 
erative diseases. Front Cell Neurosci 9:278.

4. Dahl D, Bignami A (1976) Immunogenic properties of the glial fibrillary acidic protein. Brain Res 116:150-157.

5. Schiffer D, Giordana MT, Migheli A, Giaccone G, Pezzotta S, Mauro A (1986) Glial fibrillary acidic protein and vimentin in the experimental glial reaction of the rat brain. Brain Res 374:110-118.

6. Jo S, Yarishkin O, Hwang YJ, Chun YE, Park M, Woo DH, Bae JY, Kim T, Lee J, Chun H, Park HJ, Lee DY, Hong J, Kim HY, Oh S, Park SJ, Lee H, Yoon BE, Kim Y, Jeong Y, Shim I, Bae YC, Cho J, Kowall NW, Ryu H, Hwang E, Kim D, Lee CJ (2014) GABA from reactive astrocytes impairs memory in mouse models of Alzheimer's disease. Nat Med 20:886-896.

7. Wu Z, Guo Z, Gearing M, Chen G (2014) Tonic inhibition in dentate gyrus impairs long-term potentiation and memory in an Alzheimer's disease model. Nat Commun 5:4159.

8. Woo J, Im SK, Chun H, Jung SY, Oh SJ, Choi N, Lee CJ, Hur EM (2017) Functional characterization of resting and adenovirus-induced reactive astrocytes in three-dimensional culture. Exp Neurobiol 26:158-167.

9. Roy U, Stute L, Höfling C, Hartlage-Rübsamen M, Matysik J, Roßner S, Alia A (2018) Sex- and age-specific modulation of brain GABA levels in a mouse model of Alzheimer's disease. Neurobiol Aging 62:168-179.

10. Sirevaag AM, Greenough WT (1991) Plasticity of GFAPimmunoreactive astrocyte size and number in visual cortex of rats reared in complex environments. Brain Res 540:273278.

11. van Praag H, Kempermann G, Gage FH (2000) Neural consequences of environmental enrichment. Nat Rev Neurosci 1:191-198.

12. Jung CK, Herms J (2014) Structural dynamics of dendritic spines are influenced by an environmental enrichment: an in vivo imaging study. Cereb Cortex 24:377-384.

13. Monteiro BM, Moreira FA, Massensini AR, Moraes MF, Pereira GS (2014) Enriched environment increases neurogenesis and improves social memory persistence in socially isolated adult mice. Hippocampus 24:239-248.

14. Kempermann G, Kuhn HG, Gage FH (1997) More hippocampal neurons in adult mice living in an enriched environment. Nature 386:493-495.

15. Salois G, Smith JS (2016) Housing complexity alters GFAPimmunoreactive astrocyte morphology in the rat dentate gyrus. Neural Plast 2016:3928726.

16. Woo J, Kim JE, Im JJ, Lee J, Jeong HS, Park S, Jung SY, An H,
Yoon S, Lim SM, Lee S, Ma J, Shin EY, Han YE, Kim B, Lee EH Feng L, Chun H, Yoon BE, Kang I, Dager SR, Lyoo IK, Lee CJ (2018) Astrocytic water channel aquaporin-4 modulates brain plasticity in both mice and humans: a potential gliogenetic mechanism underlying language-associated learning Mol Psychiatry 23:1021-1030.

17. Rampon C, Jiang CH, Dong H, Tang YP, Lockhart DJ, Schultz PG, Tsien JZ, Hu Y (2000) Effects of environmental enrichment on gene expression in the brain. Proc Natl Acad Sci U S A 97:12880-12884.

18. Sampedro-Piquero P, De Bartolo P, Petrosini L, ZancadaMenendez C, Arias JL, Begega A (2014) Astrocytic plasticity as a possible mediator of the cognitive improvements after environmental enrichment in aged rats. Neurobiol Learn Mem 114:16-25.

19. Ruscher K, Johannesson E, Brugiere E, Erickson A, Rickhag M,Wieloch T (2009) Enriched environment reduces apolipoprotein $\mathrm{E}$ (ApoE) in reactive astrocytes and attenuates inflammation of the peri-infarct tissue after experimental stroke. J Cereb Blood Flow Metab 29:1796-1805.

20. Chen JY, Yu Y, Yuan Y, Zhang YJ, Fan XP, Yuan SY, Zhang JC, Yao SL (2017) Enriched housing promotes post-stroke functional recovery through astrocytic HMGB1-IL-6-mediated angiogenesis. Cell Death Dis 3:17054.

21. Garofalo S, D’Alessandro G, Chece G, Brau F, Maggi L, Rosa A, Porzia A, Mainiero F, Esposito V, Lauro C, Benigni G, Bernardini G, Santoni A, Limatola C (2015) Enriched environment reduces glioma growth through immune and non-immune mechanisms in mice. Nat Commun 6:6623.

22. Robel S, Berninger B, Götz M (2011) The stem cell potential of glia: lessons from reactive gliosis. Nat Rev Neurosci 12:88104.

23. Zhang H, Muramatsu T, Murase A, Yuasa S, Uchimura K, Kadomatsu K (2006) N-Acetylglucosamine 6-O-sulfotransferase- 1 is required for brain keratan sulfate biosynthesis and glial scar formation after brain injury. Glycobiology 16:702710.

24. Sofroniew MV (2009) Molecular dissection of reactive astrogliosis and glial scar formation. Trends Neurosci 32:638-647.

25. Lee CY, Pappas GD, Kriho V, Huang BM, Yang HY (2003) Proliferation of a subpopulation of reactive astrocytes following needle-insertion lesion in rat. Neurol Res 25:767-776.

26. Martín-López E, García-Marques J, Núñez-Llaves R, LópezMascaraque L (2013) Clonal astrocytic response to cortical injury. PLoS One 8:e74039. 
27. Yoon BE, Woo J, Chun YE, Chun H, Jo S, Bae JY, An H, Min JO, Oh SJ, Han KS, Kim HY, Kim T, Kim YS, Bae YC, Lee CJ (2014) Glial GABA, synthesized by monoamine oxidase B, mediates tonic inhibition. J Physiol 592:4951-4968.

28. Lee S, Yoon BE, Berglund K, Oh SJ, Park H, Shin HS, Augustine GJ, Lee CJ (2010) Channel-mediated tonic GABA release from glia. Science 330:790-796.

29. Yoon BE, Jo S, Woo J, Lee JH, Kim T, Kim D, Lee CJ (2011) The amount of astrocytic GABA positively correlates with the degree of tonic inhibition in hippocampal CA1 and cerebellum. Mol Brain 4:42.

30. Saur L, Baptista PP, de Senna PN, Paim MF, do Nascimento P, Ilha J, Bagatini PB, Achaval M, Xavier LL (2014) Physical exercise increases GFAP expression and induces morphological changes in hippocampal astrocytes. Brain Struct Funct 219:293-302.

31. Torasdotter M, Metsis M, Henriksson BG, Winblad B, Mohammed AH (1998) Environmental enrichment results in higher levels of nerve growth factor mRNA in the rat visual cortex and hippocampus. Behav Brain Res 93:83-90.

32. Rossi C, Angelucci A, Costantin L, Braschi C, Mazzantini M, Babbini F, Fabbri ME, Tessarollo L, Maffei L, Berardi N, Caleo M (2006) Brain-derived neurotrophic factor (BDNF) is required for the enhancement of hippocampal neurogenesis following environmental enrichment. Eur J Neurosci 24:1850-1856.

33. Gobbo OL, O’Mara SM (2004) Impact of enriched-environment housing on brain-derived neurotrophic factor and on cognitive performance after a transient global ischemia. Behav Brain Res 152:231-241.

34. Bergami M, Santi S, Formaggio E, Cagnoli C, Verderio C, Blum R, Berninger B, Matteoli M, Canossa M (2008) Uptake and recycling of pro-BDNF for transmitter-induced secretion by cortical astrocytes. J Cell Biol 183:213-221.

35. Morizawa YM, Hirayama Y, Ohno N, Shibata S, Shigetomi E, Sui Y, Nabekura J, Sato K, Okajima F, Takebayashi H, Okano H, Koizumi S (2017) Reactive astrocytes function as phagocytes after brain ischemia via ABCA1-mediated pathway. Nat Commun 8:28.

36. Söllvander S, Nikitidou E, Brolin R, Söderberg L, Sehlin D, Lannfelt L, Erlandsson A (2016) Accumulation of amyloid- $\beta$ by astrocytes result in enlarged endosomes and microvesicleinduced apoptosis of neurons. Mol Neurodegener 11:38.

37. Arnaiz SL, D’Amico G, Paglia N, Arismendi M, Basso N, del Rosario Lores Arnaiz M (2004) Enriched environment, nitric oxide production and synaptic plasticity prevent the agingdependent impairment of spatial cognition. Mol Aspects Med 25:91-101.

38. Borroni E, Bohrmann B, Grueninger F, Prinssen E, Nave S, Loetscher H, Chinta SJ, Rajagopalan S, Rane A, Siddiqui A, Ellenbroek B, Messer J, Pähler A, Andersen JK, Wyler R, Cesura AM (2017) Sembragiline: a novel, selective monoamine oxidase type B inhibitor for the treatment of Alzheimer's disease. J Pharmacol Exp Ther 362:413-423.

39. de Pablo Y, Nilsson M, Pekna M, Pekny M (2013) Intermediate filaments are important for astrocyte response to oxidative stress induced by oxygen-glucose deprivation and reperfusion. Histochem Cell Biol 140:81-91.

40. Sauer H, Wartenberg M, Hescheler J (2001) Reactive oxygen species as intracellular messengers during cell growth and differentiation. Cell Physiol Biochem 11:173-186.

41. LoTurco JJ, Owens DF, Heath MJ, Davis MB, Kriegstein AR (1995) GABA and glutamate depolarize cortical progenitor cells and inhibit DNA synthesis. Neuron 15:1287-1298.

42. Chun H, Lee CJ (2018) Reactive astrocytes in Alzheimer's disease: a double-edged sword. Neurosci Res 126:44-52.

43. Qiu X, Li C, Jiang R, Chen L, Huang C, Yang S, Lu W, Shi X, Zhao Y, Gao Y, Cheng G, Tang Y (2011) The effects of shortterm enriched environment on capillaries of the middle-aged rat cortex. Neurosci Lett 505:186-190

44. Takemoto T, Ishihara Y, Ishida A, Yamazaki T (2015) Neuroprotection elicited by nerve growth factor and brain-derived neurotrophic factor released from astrocytes in response to methylmercury. Environ Toxicol Pharmacol 40:199-205.

45. Rudge JS, Pasnikowski EM, Holst P, Lindsay RM (1995) Changes in neurotrophic factor expression and receptor activation following exposure of hippocampal neuron/astrocyte cocultures to kainic acid. J Neurosci 15:6856-6867.

46. Rousseaud A, Delépine C, Nectoux J, Billuart P, Bienvenu $\mathrm{T}$ (2015) Differential expression and regulation of brainderived neurotrophic Factor (BDNF) mRNA isoforms in brain cells from Mecp2(308/y) mouse model. J Mol Neurosci 56:758-767.

47. Pang PT, Teng HK, Zaitsev E, Woo NT, Sakata K, Zhen S, Teng KK, Yung WH, Hempstead BL, Lu B (2004) Cleavage of proBDNF by tPA/plasmin is essential for long-term hippocampal plasticity. Science 306:487-491.

48. Desai NS, Rutherford LC, Turrigiano GG (1999) BDNF regulates the intrinsic excitability of cortical neurons. Learn Mem 6:284-291. 
49. Marmigère F, Rage F, Tapia-Arancibia L (2003) GABAglutamate interaction in the control of BDNF expression in hypothalamic neurons. Neurochem Int 42:353-358.
50. Khakh BS, Sofroniew MV (2015) Diversity of astrocyte functions and phenotypes in neural circuits. Nat Neurosci 18:942952. 